\title{
Das Gefängnis der Freiheit Michael Endes im Literaturunterricht.
}

\author{
Pavel Váňa
}

The article presents the collection of stories „Das Gefängnis der Freiheit" as a highly suitable source for the use in the literature class of German as a foreign language. Focussing on the stories „Einer langen Reise Ziel“ and „Das Haus an der Peripherie“ we discuss the use of cultural clichés in $19^{\text {th }}$ and $20^{\text {th }}$ century Europe. This theoretical analysis is followed by practicable methods showing how Ende's texts can be adapted for the use in the foreign language class.

Literature - cultural clichés - 19th and 20th century Europe - theoretical analysis - foreign language class

Die kurze Vorstellung von zwei Prosatexten Michael Endes Einer langen Reise Ziel und Das Haus an der Peripherie entstand mit zweierlei Absicht: zum einen soll hervorgehoben werden, dass Michael Ende bei Weitem nicht nur als ein erfolgreicher Autor der Fantasy-Literatur zu verstehen ist, zum anderen soll auf die zahlreichen Bezüge zur historischen, kulturellen, politischen und gesellschaftlichen Wirklichkeit der Zeit des ausgehenden neunzehnten und des zwanzigsten Jahrhunderts hingewiesen werden, die in Endes Sammlung von kurzen Prosatexten Das Gefängnis der Freiheit „entdeckt“ werden können.

Literaturunterricht - Deutsch als Fremdsprache - phantastische Literatur - LMS Moodle E-Kurs - literarisches Gespräch

\section{Einleitung}

Im Sommer 2009 wurde Michael Ende in seiner Vaterstadt Garmisch-Partenkirchen während zahlreicher Festveranstaltungen aus Anlass seines 80. Geburtstags als weltbekannter Autor von Fantasy-Literatur gefeiert. Um die Verankerung seines literarischen Oeuvres in dem oben genannten Bereich der Literatur deutlich in den Vordergrund zu rücken, wurde sowohl des achtzigsten Geburtstags als auch eines weiteren Jubiläums gedacht, der Herausgabe der Unendlichen Geschichte im Jahr 1979. Die Verbindung zwischen Endes Geburtstag und seinem „jubilierenden“ Werk kommt nicht von ungefähr. Ende wird heute vorwiegend als ein Kinder- und Jugendliteraturautor geschätzt. Er wird als der einzige wesentliche deutschsprachige Vertreter der Fantasy-Literatur betrachtet, die sonst vorwiegend von englischen und amerikanischen Autoren und Autorinnen ,beherrscht' wird.

In diesem Beitrag möchte ich mich auf einige literarische Texte Michael Endes konzentrieren, die gewöhnlich nicht der Fantasy-Literatur zugeordnet, sondern als fantastische Literatur betrachtet werden. Die Trennungslinie zwischen Fantasyund fantastischer Literatur wird anhand der einschlägigen Definitionen Gero von 
Wilperts gezogen. ${ }^{1}$ An einigen kurzen Prosatexten Michael Endes soll dokumentiert werden, dass die für die erwachsenen Rezipienten bestimmten Prosawerke Michael Endes für die Arbeit in einem literarischen Seminar des Faches DaF sehr wohl verwendet werden können.

\section{Literarische Werke Michael Endes für den erwachsenen Leser}

Seit Mitte der achtziger Jahre lenkte M. Ende sein literarisches Augenmerk zunehmend auf den erwachsenen Leser. Dabei bedient er sich bei der Verfassung der Werke für den erwachsenen Leser einer Vielfalt von literarischen Gattungen: In rascher Folge entstanden u. a. das Theaterstück Das Gauklermärchen (1982), Sammlungen von kurzen Prosatexten Der Spiegel im Spiegel (1983) und Das Gefängnis der Freiheit (1992), eine Sammlung von lyrischen Texten Trödelmarkt der Träume (1986), Libretti zu den Opern Der Goggolori. Eine bairische Mär (1984) und Der Rattenfänger. Ein Hamelner Totentanz (1993).

Eine tiefere Einsicht in die Kindheit und Jugend von Michael Ende, aber auch in die Jahre seiner ersten literarischen Erfolge und seines langen Aufenthalts in Italien, der u. a. aus seiner Enttäuschung über die Ungunst der deutschen Literaturkritik seinen Werken gegenüber resultierte, gewähren zwei Memoirenbücher - Edgar Ende (1971) und Die Archäologie der Dunkelheit (1985). Das Letztere, ein Interview, das Michael Ende 1985 während seines Italien-Aufenthalts Jörg Krichbaum gegeben hat, umfasst vier Tage von Gesprächen und Reflexionen über Michael Endes Vater, Edgar Ende, über dessen Empfinden der Welten außerhalb der sinnlich erkennbaren Realität, dessen Beziehung zur surrealistischen Kunst und dessen eigene ,Konstruktion' oder ,Architektur' von Bildern. Michael Ende wendet sich wiederholt gegen die Versuche, seinen Vater als einen surrealistischen Maler zu interpretieren ${ }^{2}$

1 Nach Gero von Wilpert ist die Fantasy-Literatur eine Literatur, die „ [...] die Phantasie mit gänzlich imaginären, [...], archaischen Alternativwelten und -zeiten mit magisch-mythischen Wesen ohne Naturwissenschaft und Technik und mit Pseudomythologien u. ä. evozieren will [...].“ (Wilpert 2001, 260). Die phantastische Literatur ist laut Wilpert ein „Sammelbegriff für alle Literatur außerhalb religiös mythischen Kontexts, die die realistische Ebene überschreitet zugunsten des Irrealen, Surrealen, Wunderbaren, Übernatürliche, Zauberhaften, Unheimlichen, Bizarren, Grotesken, Okkulten, Traumhaften, Unbewußten, Halluzinatorischen, Visionären, GespenstischGeisterhaften [...]. Sie geht dabei oft vom Realen aus und eröffnet plötzlich oder allmählich eine phantastische Gegenwelt, die die Realität verfremdet und übernatürliche Mächte und Wesenheiten postuliert [...]." (ebd., 607).

2 Auf die Frage Krichbaums nach der Beschäftigung Edgar Endes mit esoterischen Schriften erklärt M. Ende, für seinen Vater gäbe es „keinen Zweifel daran, daß es hinter der Welt der sinnlichen Wahrnehmungen eine oder viele andere Welten gibt, die wir zwar mit den Sinnen nicht wahrnehmen können, die aber ebenso wirklich sind [...]. [...] die Kunst stellte sich ihm als Brücke dar [...] zu dieser anderen Welt. Und die [...] überraschenden Zusammenfügungen oder diese scheinbar paradoxen Zusammenfindungen auf seinen Bildern [...] resultierten für ihn aus der Überzeugung, daß in den geistigen Welten die Dinge sich [...] ganz anders darstellen und daß man diese [...] nur durch solche merkwürdigen Zusammenfindungen erreichen konnte“ (ENDE: 1985, 40). 
und deutet an, dass die von Edgar Ende gemalten Bilder - seine Aufzeichnungen eigenartiger Visionen aus dem Bereich des sinnlich nicht Wahrnehmbaren - als eine der wichtigsten Inspirationsquellen für sein literarisches Schaffen anzusehen seien.

\section{Das Gefängnis der Freiheit}

In Michael Endes Spätwerk Das Gefängnis der Freiheit tauchen die Fragen seines Vaters nach Stellen, Orten und Räumen der durch sinnliche Wahrnehmung unerreichbaren Welten auf und verbinden sich mit Michael Endes Suche nach einer künstlerischen Erfassung dieser vermuteten geistigen Welten in Worten, nach ihrer literarischen Darstellung.

In acht Geschichten, die allesamt esoterisch (im Sinne geheimnisvoll, diffus, nebulös) anmuten, bewegt sich Ende auf einem Terrain, das sich von leichter Parodie bis zum Grotesken erstreckt. Seine Aufmerksamkeit gilt den prägenden Details, die zu bestimmten zeitlichen, räumlichen oder kulturellen Phänomenen in Beziehung gesetzt werden und dadurch zur Charakteristik einer geschichtlichen Epoche bzw. einer nationalen oder lokalen Eigenart verhelfen. Um sie in den Vordergrund zu rücken, bedient sich M. Ende zahlreicher Topoi.

\subsection{Das literarische Spiel mit dem Raum}

Die räumliche Einordnung der Handlung geschieht in der Sammlung kurzer Geschichten Das Gefängnis der Freiheit auf dreierlei Weise:

A: Es wird ein konkreter Ort genannt und durch verschiedene Topoi deutlich charakterisiert.

Diese Art örtliche Bestimmung findet man in der ersten (Einer langen Reise Ziel) und der letzten Geschichte (Die Legende vom Wegweiser) des Bandes. In Einer langen Reise Ziel bilden vorwiegend England und indirekt die Weltdimension des viktorianischen Imperiums, Deutschland und Italien die räumliche Kulisse. In beiden o. g. Geschichten versucht der Protagonist, einen neuen Ort zu finden, der eine besonders starke Anziehungskraft auf ihn ausübt. Das Erreichen dieses Ortes bedeutet für die Hauptfigur die Erfüllung ihrer lebenslangen Sehnsucht.

B: Es wird zwar ein konkreter Ort genannt, aber wenn man an diesen Ort gelangt, erscheint man in einer neuen, befremdlichen Dimension, die keine nähere lokale Bestimmung erfährt und den Anschein einer räumlich „unendlichen“ oder „unbegrenzten“ Dimension erweckt.

$\mathrm{Zu}$ dieser Gruppe sind vor allem die Geschichten Der Korridor des Borromeo Colmi, Das Haus an der Peripherie und Zugegeben etwas klein zu zählen.

C: Die Grenzen der Handlungsorte verschwimmen völlig, die Handlung spielt sich in Räumen ohne abgegrenzte Räumlichkeit ab. 
In den Geschichten dieser Gruppe kommen keine genauen geographischen Ortsangaben vor. Diese Art Raumangabe soll alles Konkrete, Geographische ausblenden und dadurch den Zeit- und/oder Ortzusammenhang zerstören. Die Geschichte spielt sich dann in einer archetypisch erscheinenden Gegend ab. Einmal ist es die Wüste mit einer geheimnisvollen Stadt, wobei es sich eigentlich um eine der unzähligen Traumwelten handelt, die wir hinter uns lassen (Aus den Aufzeichnungen des Traumweltreisenden Max Muto), ein anderes Mal wird der Handlungsort noch verschwommener definiert (Das Gefängnis der Freiheit).

\subsection{Das Klischee als ein Raum- und Zeitcharakteristikum: Einer langen Reise Ziel}

Die Art und Weise, wie Ende in seinen Erzählungen von verschiedenen Klischees Gebrauch macht, lässt sich am Beispiel der Geschichte Einer langen Reise Ziel verdeutlichen. In der Geschichte spielt M. Ende mit einer Vielzahl von Topoi, die das England der viktorianischen Epoche bzw. das wilhelminische Deutschland, das Osmanische Reich oder das Italien dieser historischen Epoche betreffen. Am Beispiel der Legenden- und Volksbuchfigur des ,Ewigen Juden' Ahasveros wird die klischeehafte Vorstellung von einem jüdischen Weisen verdeutlicht, der das ,Geheimnisvolle' bei Juden symbolisiert.

Mit überspitzter Ironie konstruiert M. Ende Cyril Abercomby, den Protagonisten der Geschichte, als den Prototyp eines überkultivierten englischen Adeligen. Bei der Charakterbeschreibung Cyrils wird - bereits in seiner Kindheit - ein starrer, dem Leben abgewandter Gesichtszug festgestellt und für das Gesamtbild dieses Helden wird konsequent eine abstoßende Wirkung intendiert:

Cyril war kein Kind, das Sympathie erweckte [...]. Er war [...] von einem knochigen, sozusagen fleischlosen Körperbau, hatte strohige, farblose Haare, wässrige, ein wenig vorquellende Augen [...]. Am merkwürdigsten aber für einen Jungen seines Alters war der völlige Mangel an Bewegung in seinem Gesicht. (ENDE: 2002, 8)

Nicht nur am äußerlichen Aussehen Cyrils, auch an seinen Taten kommt seine Indolenz, „kühle Höflichkeit“ (ebd., 9) zum Vorschein. In den Schlüsselsituationen der Erzählung zeigt er seinen festen Willen, der keinen Widerspruch zulässt und sich in seinem Handeln als überhebliche Arroganz manifestiert. Die (ausschließlich im erotischen Bereich praktizierte) Liebesbeziehung Cyrils zu Isolde, der Tochter eines deutschen Bankiers, aber auch eine ähnlich erotisch basierte Liebesbeziehung seines Erziehers Mr. Ashleys zu Cyrils Erzieherin Miss Twiggle, zeichnet sich durch berechnende Skrupellosigkeit und tiefste Verachtung der Frau gegenüber aus. Beide Frauen werden als für ihre Partner unerträgliche, naive Wesen geschildert, in deren 
Gedankenwelt Liebe und Beziehung mit absoluter Hingabe gleichgesetzt wird. Die moralische ,Rettung' des geliebten Mannes erheben sie zu ihrer wichtigsten Lebensaufgabe. Eine Frau, die es ablehnt, diese im 19. Jahrhundert übliche Frauenrolle passiv zu spielen, muss scheitern (so z. B. Cyrils Mutter).

Nicht nur die einzelnen Figuren, sondern auch das Gesamtbild der englischen viktorianischen Gesellschaft werden im grotesken Stil geschildert. Das Letztere ist z. B. der Fall, als Cyril nach dem Tod seines Vaters verfügt, den geerbten Besitz der Familie Abercomby zu verkaufen:

Als die Öffentlichkeit davon erfuhr [...] brach ein Sturm der Entrüstung los. Nicht nur der Hochadel [...] brachte einhellig größten Abscheu vor einem so unerhörten Mangel an Traditionssinn und Standesbewusstsein zum Ausdruck [...], ja, sogar in den Pubs der unteren Schichten gab es [...] erhitzte Diskussionen über die Frage, ob ein solcher Mensch [...] noch das Recht habe, sich Untertan Ihrer Majestät zu nennen. Juristisch bestand jedoch keinerlei Handhabe gegen diesen „Ausverkauf englischer Kultur und Würde“ [...]. (ENDE: 2002, 27)

Als sich aber die ersten amerikanischen Käufer melden und einen Teil des Erbes abkaufen, „war es wie ein Schock für den nationalen Stolz. Um zu retten, was noch zu retten war, begann ein wahrer Run seitens der reichen [...] Familien des Empires auf alles, was noch zu haben war" (ebd., 29). Auch an vielen anderen Stellen dieser Erzählung geht M. Ende in spielerischer Manier mit den Klischees um, die auf die einzelnen (nicht nur) europäischen Länder Bezug nehmen. Eine Gelegenheit dazu bietet sich, als Cyril auf Einladung des Kommerzienrats Jakob von Erschl nach Frankfurt am Main kommt. Der Deutschlandbesuch wird im Hotel „Zum Römer" eröffnet, wohin Cyril zu einem „Dinner im kleinen Kreise einiger Kunstund Musikfreunde" (ebd. 32) eingeladen wird. Die Tochter des Kommerzienrats und Cyrils künftige Geliebte, Fräulein Isolde, „ein rundliches junges Mädchen mit Gretchenfrisur" (ebd. 32), singt einige Lieder, wobei Frau Kommerzienrätin ein Gespräch mit Cyril einzufädeln versucht:

Wir Deutschen [...] sind nun einmal das Volk, das alle wahrhaft großen Komponisten hervorgebracht hat. Selbst Händel, den ihr Engländer ja für euch in Anspruch nehmt, stammte aus Deutschland. Das müssen Sie zugeben, Mylord. (ebd., 32)

Die deutsch-englische Rivalität um die Vormachtsstellung der wilhelminischen (bzw. viktorianischen) Epoche wird an dieser Stelle der Erzählung mehrmals zwischen den handelnden Figuren ausgetragen. Wenn Jakob von Erschel nicht bereit ist, Cyril ein Bild aus seiner Sammlung zu verkaufen, für das Cyril immer höhere Summen anbietet, erklärt der Kommerzienrat seiner Frau: 
Manche Engländer [...] glauben wohl, dass es unsereinem nur ums Geld zu tun ist. Das mag für das perfide Albion zutreffen, aber bei uns in Deutschland gibt es noch den Glauben an Ideale. [...] Dieser arrogante Brite soll lernen [...], daß man für Geld nicht alles [...] kaufen kann. (ebd., 40)

Um dem antienglischen Akzent Nachdruck zu verleihen, macht Ende an dieser Textstelle von der Antonomasie,Albion' für ,England' Gebrauch: Versehen mit dem personifizierenden Attribut ,perfide' wird an eine Bezeichnung Englands erinnert, die in der erzählten Zeit dieser Geschichte nicht unbekannt war. Heute erscheint diese Verbindung als völlig unakzeptabel. Deshalb müsste sie bei einer eventuellen Besprechung im Literaturunterricht zunächst auf eine entsprechende Weise erklärt werden. Dann wäre argumentierend zu zeigen, dass ihre Verwendung im Text zweifellos auf eine andere Erzählabsicht hinweist, als England gegenüber ausfallend zu werden.

Klischeebeladen sind die Beschreibungen überall dort, wo die Existenz eines das jeweilige Land prägenden Phänomens festgestellt wird. Das ist z. B. der Fall, als Cyril einen der Direktoren der Bank von England in London besucht (vgl. ebd., 45) oder an der Stelle der Erzählung, an der der Leser die Figur eines italienischen international renommierten Kunstdiebes näher kennen lernt. Der Ort der Handlung ist Italien, daher tauchen zur Abwechslung einige Topoi auf, die mit Italien verbunden sind:

Er professore war ein kleiner, höchst agiler Mann um die Vierzig mit frauenhaft zarten Händen [...]. Er lebte in einer prächtigen Villa, in der seine ganze weit verzweigte Familie auf irgendeine Art angestellt war. Zum Kreise seiner Kunden und Gönner gehörten außer einigen bedeutenden Camorristen auch ein paar Minister und Kardinäle [...]. Die Polizei hielt sich ihm gegenüber dementsprechend zurückhaltend in ihren Nachforschungen. (ENDE: 2002, $57-58)$

\subsection{Die Räume, die es nicht gibt: Das Haus an der Peripherie}

Mit länderspezifisch gefärbten Klischees arbeitet $M$. Ende auch in anderen Erzählungen der Sammlung Das Gefängnis der Freiheit. Auf der allgemein verbreiteten Vorstellung einer typisch italienischen Familie mit einer beachtlichen Anzahl von Familienmitgliedern und ihrer deutlich patriarchalischen Ausprägung gründet Endes Geschichte Zugegeben etwas klein. Er entwickelt ein ad absurdum geführtes Spiel mit Räumen, die es in Wirklichkeit nicht geben kann. Während der Ich-Erzähler dieser Geschichte von der Existenz mehrerer Räume zunächst nichts ahnt und sie dann verwundert zur Kenntnis nehmen muss, wird in der Geschichte Das Haus an der Peripherie die Frage der Raumexistenz auf eine radikal umgekehrte Weise behandelt: Den Raum, dessen Vorhandensein automatisch vorausgesetzt werden müsste, gibt 
es in Wirklichkeit nicht. Das überraschende Fehlen des Raumes - das Innere eines relativ großen Hauses - wird mit der Leere des nationalsozialistischen Regimes verglichen und der Wertlosigkeit jeder Diktatur gleichgesetzt.

Die Geschichte ist als Leserbrief des Studienrats a. D. Dr. phil. Joseph Remigius Seidl an Michael Ende stilisiert. Der Autor des Briefes erzählt, wie er 1942 als elffähriger Junge zusammen mit seinem Bruder versuchte, das Geheimnis eines Hauses aufzudecken, das kein Inneres hatte. Beide Jungen unternahmen mehrere Versuche in das Haus zu kommen, um schließlich festzustellen, dass es keine Innenräume hat, obwohl es von außen wie ein relativ großes viereckiges Haus aussah, das „den Stil jener öden Pseudoklassizistik [zeigte], der für alle Diktaturen unseres Jahrhunderts, gleich ob faschistische oder sozialistische, kennzeichnend ist" (ENDE: 2002, 101).

Die Figurenkonstellation dieser Geschichte ist einfach, sie übt jedoch eine starke Wirkung aus. Zwei Jungen, 11 und 14 Jahre alt, und eine ältere Frau, die einzige Person, die das Haus ohne Innenräume auf eine unbegreifliche Weise regelmäßig als Putzfrau betreten hat. Die innere Spannung dieser Konstellation entsteht durch den Kontrast zwischen der naiv direkten, abenteuerlustigen, ,normal' kindischen Wahrnehmungder Knaben, für die eine Nicht-Existenzdes Innenraumes unbegreiflich ist, und der ominösen, eigenartigen, frostig distanzierten Frau. Die Knaben können die Entdeckung der Leere des Hausinneren nicht endgültig akzeptieren. Sie fragen, experimentieren, untersuchen, provozieren. Völlig anders zeigt sich die Frau, die wegen der "Wolke von schwerem Gestank, die sie meistens umgab“ (ENDE: 2002, 97) ,Schoaßwalli' genannt wurde. Für sie sind die nicht vorhandenen Innenräume des Hauses ein vertrautes Milieu. Im Unterschied zu den zwei Jungen kommt alles, was sich auf sie bezieht, aus dem Bereich des Geheimnisvollen, Zwielichtigen, nur andeutungsweise Offenbarten. Sie spricht oder fragt meistens nur mit dem Ziel zu zeigen, dass sie vielleicht mehr weiß, als man ahnt. Diese Andeutungen helfen ihr, ihre Umgebung zu manipulieren und den Menschen Angst einzujagen. Die Erwachsenen können aufgrund ihrer eigenen Erfahrung mit dem Bösen ziemlich schnell eine vorsichtige Haltung gegenüber der Schoaßwalli einnehmen. Der Autor des ,Leserbriefs' erinnert sich an ein unbeabsichtigt gehörtes Gespräch zwischen seiner Mutter und ihrem Bruder:

Einmal schnappte ich [...] eine Bemerkung [...] des Inhalts [auf], daß man sich vor der Schoaßwalli besser in acht nehme. [...] Es gäbe da einen gewissen okkulten Kreis, [...] der die Ankunft einer außer- oder unterirdischen Rasse von Übermenschen vorbereite. (ebd., 98-99)

Die Erwachsenen legen der Schoaßwalli gegenüber eine klare Distanz an den Tag: 
Über die wahre Identität dieser Dame konnte ich bis heute nur wenig in Erfahrung bringen. Die meisten Leute, die in Feldmoching etwas mit ihr zu tun hatten, wollten mir keine Auskunft geben oder leugneten einfach, sie gekannt zu haben. (ebd., 98)

Die Jungen sind einer Distanz gegenüber dem geheimnisvollen Bösen, wie sie die Erwachsenen an den Tag legen, zunächst nicht fähig. Die Schoaßwalli ist für sie nur eine skurrile, Neugier erweckende Figur. Am Anfang symbolisierte die Frau etwas Abenteuerliches, sich von der Alltäglichkeit des Ortes Abhebendes, und die Jungen hegen einige Sympathien für sie: „Was allerdings unser knabenhaftes Interesse am meisten erregte, war der Umstand, daß sie sich offensichtlich niemals wusch. Ihre langen Fingernägel starrten vor Schmutz, ihr Hals und ihr Gesicht waren streifig" (ebd., 97). Selbst den Knaben wird aber bald klar, dass die Schoaßwalli vielmehr als Symbol für etwas Unheimliches steht. Sie werden zufällig Zeugen einer Szene, in der diese Vermutung zur Gewissheit wird:

Einmal - das wird wohl schon gegen Ende 1943 gewesen sein - kam ein schwarzer Mercedes, in dem mehrere Männer saßen. Der Wagen wartete über eine Stunde auf der Straße vor dem Haus, bis die Schoaßwalli auf ihrem Fahrrad eintraf. Zwei SS-Männer in Uniform stiegen aus dem Auto, zwischen sich führten sie einen Mann in Hut und Mantel. Er war sehr bleich. Die Dame übernahm den Gefangenen - jedenfalls hielten wir ihn für einen solchen - , und er folgte ihr willenlos ins Haus. Nach einer Weile kam sie allein zurück, die SS-Leute grüßten mit dem, was man damals den deutschen Gruß nannte, sie erwiderte die Geste und radelte fort. (ebd., 109-110)

Die Knaben haben begriffen, dass es im Unterschied zu Walli Leute gibt, für die das Betreten des Hauses eine Ausweglosigkeit im wahrsten Sinne des Wortes bedeutet. Nicht nur die Frau, sondern auch das Haus werden ziemlich eindeutig mit der nationalsozialistischen Ära identifiziert (die strenge Baustruktur des Hauses, Swastika-Bilder an dessen Außenwänden). Die Wahrheit über das Haus erfahren die Knaben im Traum:

[...] oft träumten wir von dem Haus, einige Male sogar gleichzeitig den selben Traum [...]: Wir standen nahe [...] dem Haus. [...] nach und nach gewahrten wir ein leichtes Vibrieren des Bodens, das immer zunahm, so als stünden wir auf dem Fell einer unermeßlich großen Trommel, welches in der Resonanz auf einen unterirdischen oder infernalischen Klang mitschwang [...]. Gleichzeitig erschien hinter den Fensterschlitzen im Inneren des Hauses ein übergrelles [...] Licht [...]. Das überaus Entsetzliche an diesem tödlichen Licht dort drin war einfach die Tatsache, daß es existierte. Wir beide empfanden [...], daß es die 
Präsenz von etwas anzeigte, das man nicht anders nennen kann als: das absolute Böse. (ebd., 108-109)

Diese Erkenntnis beendet die Zeit der sorglosen Kindheit beider Knaben. Der ältere von ihnen bleibt am Ende des Krieges von dem „absolut Bösen“ nicht verschont: Er stirbt „eben sechzehnjährig, zur Wehrmacht eingezogen [...] an der Ostfront, die ja zu diesem Zeitpunkt bereits in heilloser Auflösung war" (ebd., 113).

Der Entwurf der Geschichte als eines fiktiven Leserbriefes aus dem Jahr 1985 ermöglicht dem Autor den Brief mit einer zeitbezogenen Notiz zu beenden, die nicht nur vierzig Jahre nach dem Ende des Zweiten Weltkriegs aktuell ist:

Daß die Existenz jenes Hauses [...] heute so unbeweisbar und so unglaublich erscheint, als wäre es nie gewesen, fügt sich [...] nur allzu gut ins Bild der Zeit. Nicht anders verhält es sich ja mit so manchem in unserer jüngsten Geschichte. (ebd., 116)

Der unkomplizierte Schreibstil M. Endes ist nicht das Ergebnis einer mangelhaften Erzählkunst, sondern ein Programm, das der Autor mehrmals verkündet hat. In dem Interview-Buch Die Archäologie der Dunkelheit findet man diesen schriftstellerischen Grundsatz M. Endes bestätigt:

Ich [bin] eigentlich kein Literat. Im Gegenteil, mich [...] ärgern gewisse stilistische Koketterien bei Schriftstellern [...]. Ich habe dabei oft das Gefühl, als ob mich der Autor mit hochgezogenen Augenbrauen ansieht und zu mir sagt: Hast du auch gemerkt, wie fein ich das wieder formuliert habe? [...] Ich will das nicht. Ich finde, der epische Stil muß transparent sein. [...] Ich möchte nicht immerfort auf den Erzähler hingewiesen werden. Ich möchte die Erzählung hören. (ENDE: 1985, 134)

\section{Endes literarische Texte im Unterricht}

Die klare Symbolik, die Transparenz der Absicht, mit der die oben erwähnten Topoi/ Klischees (kon)textualisiert werden, die deutlich erkennbaren Erzähltechniken und Verfahren der literarischen Fiktion machen die Texte in dem Erzählband Das Gefängnis der Freiheit attraktiv für den Literaturunterricht in den Seminaren des Hochschulstudiums DaF. Abschließend möchte ich daher einen kurzen Unterrichtsentwurf skizzieren: Dabei werden einige Strategien der Texterschließung im Literaturseminar des Faches DaF vorgeführt. Um bei einer kurzen Probe bleiben zu können, werde ich mich nur den nicht schriftlichen Verfahren der Interpretation zuwenden. 
Als die traditionelle Form einer nicht schriftlichen Interpretation wird nach wie vor das gelenkte Gespräch des Lehrers mit den Lernern in einem Literaturseminar angewendet. Als Gegenentwurf dazu kann das literarische Gespräch angesehen werden. Hierbei beteiligt sich der Lehrer nur initiierend und moderierend an dem Gespräch. In Fachkreisen (u. a. MERKELBACH: 1998; IVO: 1994; WERNER: 1996) wird in der letzten Zeit die Frage diskutiert, ob die „strukturierende[n] und lenkende[n] Eingriffe" (ABRAHAM/KEPSER: 2009, 233) des Lehrers das literarische Gespräch beleben oder es eher behindern und im Extremfall sogar zum Stillstand bringen können.

Es ist wichtig, dass die Lehrkraft - besonders bei den ersten Versuchen einer Textinterpretation im Literaturunterricht - das literarische Gespräch mit Hilfe von vorgegebenen Fragestellungen oder einer empfohlenen Strukturierung des Gesprächs lenkt und kreativ/improvisatorisch einen Wendepunkt im Gespräch initiiert, wenn es nicht konstruktiv verläuft.

Bei der Vorbereitung des literarischen Gesprächs kann man - je nach den gegebenen Bedingungen variierend - auf das von Spinner (vgl. SPINNER: 2006, 14) präsentierte Schema der sechs Phasen eines literarischen Gesprächs zurückgreifen. Da dieses Schema ursprünglich nicht für den Hochschulunterricht gedacht ist, kann man die Unterrichtseinheit des Literaturseminars, in der das Gespräch stattfinden soll, als eine thematische Einheit in einem E-Learnig-Kurs konzipieren. Bei dieser Lösung wird das Thema eines E-Kurses (der z. B. im LMS Moodle ${ }^{1}$ angelegt wird) gezielt für den Zweck des literarischen Gesprächs gestaltet. Die Verwendung der interaktiven Module des Moodle-Themas ermöglicht es, dass der Lerner als eines der Rezeption begleitenden Verfahren bereits in der Vorbereitungsphase an den Lehrer bzw. die anderen beteiligten Lerner Fragen richten kann, die sich auf das vorgegebene literarische Werk, das besprochene Thema, ggf. das beabsichtigte Ziel des literarischen Gesprächs beziehen. Durch die Gestaltung eines begleitenden Themas im E-Learning-Kurs kann der Punkt 3 („der Text wird [in der Unterrichtseinheit] zunächst vorgetragen, dann ausgeteilt und noch einmal still gelesen“), eventuell auch der Punkt 4 (,die leitende Person gibt durch einen anregenden Impuls allen die Gelegenheit zu einer ersten Äußerung") des von Spinner vorgeschlagenen Schemas entfallen (SPINNER: 2006,14). Die anregenden Impulse können bereits im E-Kurs-Thema formuliert werden. Dies würde sicher zu einem effizienteren Gesprächsverlauf bei der Texterschließung führen.

1 Das Learning-Management-System Moodle ist eine offene Lernplattform, die durch ihr benutzerfreundliches Softwaresystem eine kreative, lernzielgerechte Gestaltung von unterrichtsbegleitenden Online-Kursen ermöglicht. Durch eine Reihe von Modulen (Vorlesung, Übungen, Buch, Textseite) können die Inhalte abwechslungsreich sowohl von der Lehrkraft, als auch in Zusammenarbeit mit den Lernern bearbeitet und weiter entwickelt werden. Die interaktiven Module des LMS Moodle - Forum, Chat, Dialog - ermöglichen einen durchgehenden Kontakt zwischen dem Lehrer und Lerner oder zwischen den Lernern untereinander. 
Die Erzählungen Michael Endes aus der Sammlung Das Gefängnis der Freiheit können wegen ihrer Vielschichtigkeit und zugleich ihrer gestalterischen Transparenz für die oben kurz erwähnte Arbeitsweise in einem Literaturseminar effektiv herangezogen werden. Bei der Besprechung dieser Texte im Literaturunterricht erschließen sich Fragen aus vielen fachübergreifenden Gebieten: der Geschichte, Landeskunde, Soziologie, Psychologie, Philosophie u. a.

Die Lehrkraft kann zu den oben erwähnten Geschichten Michael Endes Fragen an die Lerner richten (entweder im voraus als eine vom Lehrer erstellte Unterrichtscollage im Moodle-Kurs oder im Verlauf der Unterrichtseinheit, die diesem Thema gewidmet ist), die den Lerner dazu motivieren, sich zusätzliche Kenntnisse über die politische, wirtschaftliche und kulturelle Entwicklung in der zweiten Hälfte des 19. Jahrhunderts zu beschaffen und sich eingehender mit der Problematik der Beziehung zwischen den europäischen Großmächten der betreffenden Epoche zu beschäftigen.

Dieses Vorwissen kann bei der Besprechung der Geschichte Das Haus an der Peripherie Verwendung finden: Der Lerner kann den Widerstreit der Interessen einzelner europäischer Länder/Kulturräume als eine kontinuierliche Linie verfolgen, die sich zeitlich vom 19. bis in die Mitte des 20. Jahrhunderts zieht. Auf Grund dessen gewinnt er eine komplexere Einsicht in die Logik der Entwicklung in Deutschland nach 1918. Nicht nur die chronologische Folge einzelner historischer Ereignisse sollte das Fragenkompendium betreffen. Das Haus an der Peripherie kann aus mehreren Blickwinkeln betrachtet und analysiert werden:

- der Nationalsozialismus als ein Teil des Extremismus, seine Spezifika und seine Ähnlichkeit mit anderen extremistischen Regierungsformen (Kommunismus u. a.);

- der Mechanismus und die Funktion der Angst in einem totalitären Regime;

- die Gleichgültigkeit gegenüber dem Inneren, die Wahrnehmung des Äußeren;

- die fehlende Solidarität (zwischen einzelnen Menschen, zwischen Generationen u. a);

- die politische, kulturelle, gesellschaftliche etc. Situation im Europa der Zwischenkriegszeit;

- die Weltvorstellung eines Kindes;

- die Frage der (Mit)Schuld, die Art der (durchaus nicht nur deutschen) Auseinandersetzung mit der Vergangenheit.

In den interaktiven Modulen des E-Kurses kann die Ausarbeitung einzelner Problemkreise in Teamarbeit erfolgen. Der Vorteil einer solchen Herangehensweise für die Lerner ist die Möglichkeit, die für sie am interessantesten Fragestellungen in den Vordergrund zu rücken und sich (nach eventueller Absprache mit der Lehrkraft) im Seminar vorwiegend auf bestimmte ausgewählte Ebenen des literarischen Werkes zu konzentrieren. 
Dabei sind die literaturwissenschaftlichen Aspekte (der Textstruktur, Figurenkonstellation, Erzählsituation, Erzählperspektive u. s. w.) als ein nicht wegzudenkender Teil des Gesprächs, ggf. auch als E-Kurs-Thema zu betrachten. Michael Endes Werk ist dermaßen vielschichtig und vieldeutig, dass sich damit eine Reihe von Möglichkeiten für den Einsatz im Literaturunterricht ergibt. Über die konkrete Gestaltung einer Literaturstunde, in der die oben genannten Texte von Ende behandelt werden, möchte ich in einem anderen, literaturdidaktisch aufgefassten Beitrag referieren.

\section{Literatur:}

ENDE (2002): Ende, Michael. Das Gefängnis der Freiheit. München: Goldmann, 2002.

ENDE (1985): Ende, Michael. Die Archäologie der Dunkelheit: Gespräche über Kunst und das Werk des Malers Edgar Ende. Stuttgart: K. Thienemanns, 1985.

ABRAHAM (2009): Abraham, Ulf/Kepser, Matthis. Literaturdidaktik Deutsch. Eine Einführung. Berlin: Erich Schmidt, 3. Aufl., 2009.

IVO (1994): Ivo, Hubert. Muttersprache - Identität - Nation. Sprachliche Bildung im Spannungsfeld zwischen einheimisch und fremd. Opladen: Westdeutscher Verlag, 1994.

MERKELBACH (1995): Merkelbach, Valentin. Zur Theorie und Didaktik des literarischen Gesprächs, in: Christ, Hannelore et al. (Hg.) "Ja aber es kann doch sein... "In der Schule literarische Gespräche führen. Frankfurt am Main: Lang, 1995, 12-52.

MERKELBACH (1998): Merkelbach, Valentin. Über literarische Texte sprechen, in: Der Deutschunterricht, 1998, 1, 74-82.

SPINNER (2006): Spinner, Kaspar H. Literarisches Lernen, in: Praxis Deutsch, 2006, 33, Nr. 200, 6-16.

WERNER (1996): Werner, Johannes. Literatur im Unterrichtsgespräch - Die Struktur des literaturrezipierenden Diskurses. München: Vögel, 1996.

WILPERT (2001): Wilpert, Gero von. Sachwörterbuch der Literatur. Stuttgart: Alfred Kröner, 8. Aufl., 2001. 